\title{
High voltage RF generator and compensation network for a FFT ion trap mass spectrometer
}

\author{
Michael Schmidt ${ }^{1}$, Albrecht Brockhaus ${ }^{1}$, Stefan Butzmann ${ }^{1}$, Michel Aliman ${ }^{2}$ \\ ${ }^{1}$ University of Wuppertal, Germany \\ ${ }^{2}$ Carl Zeiss SMT GmbH, Oberkochen, Germany
}

\begin{abstract}
In this work we present two electronic key components to operate a quadrupole ion trap mass spectrometer. Radio frequency high voltage generation is mandatory to create a trapping field. Our trap works in the Fourier transform mode, consequently very small induction currents have to be detected from the ion motion. Since interference signals from the storage voltage are many orders of magnitude higher than the detection signal, a sophisticated compensation circuit is required at the amplification stage.
\end{abstract}

Index Terms-Low-noise amplifiers, Signal generators, Mass spectroscopy

\section{INTRODUCTION}

The cylinder-symmetric quadrupole ion trap consists of three hyperbolically shaped electrodes, see Figure 1. By applying an alternating voltage to the ring-electrode while keeping the end-cap electrodes at ground potential, ions can be successfully stored in the trap.

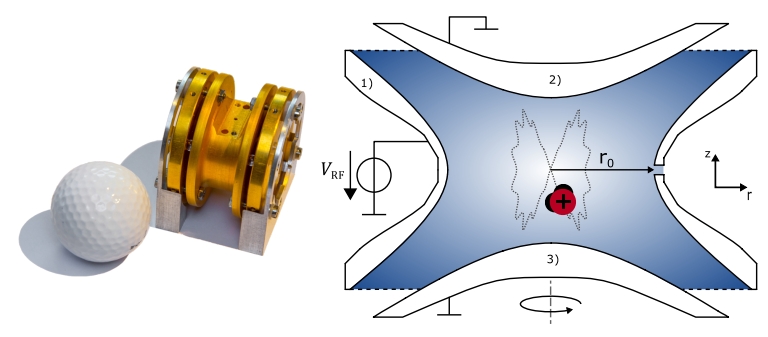

Fig. 1. Quadrupole ion trap, golf ball for size comparison. 1) ring electrode, 2) top end-cap electrode, 3 ) bottom end-cap electrode (ion trajectory is outlined)

Ions oscillate with a characteristic resonance frequency depending on their mass-to-charge ratio $\mathrm{m} / \mathrm{z}$. The value of the ion frequency for oscillation in $\mathrm{z}$ direction is given by

$$
f_{\mathrm{z}_{\text {ion }}}=\beta \frac{f_{\mathrm{RF}}}{2},
$$

where $f_{\mathrm{RF}}$ is the frequency of the trapping voltage $V_{\mathrm{RF}} \cdot \beta$ is called the stability parameter and can be derived by solving the differential equation of ion motion. Ion oscillations become unstable for $\beta \geq 1$. For values of $\beta<0.3$ we have

$$
\beta \propto \frac{V_{\mathrm{RF}} z}{m f_{\mathrm{RF}}^{2}} .
$$

In our research one goal was to conduct broadband ion measurements (one mass spectrum per shot). The other goal was to enlarge the mass range to the

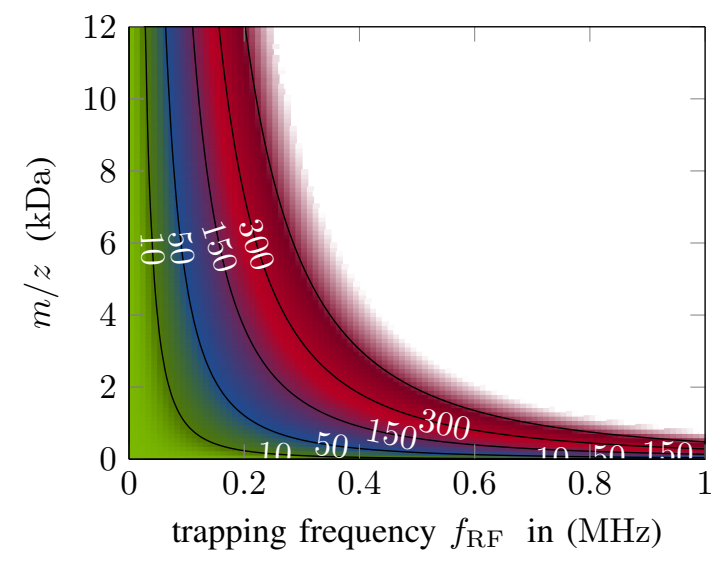

Fig. 2. Required amplitude $V_{\mathrm{RF}}$ in volt to obtain the desired mass range with a given trapping frequency

$\mathrm{kDa}$ range. The latter may be achieved by increasing the amplitude of the applied storage field. In this case, several $\mathrm{kV}$ would need to be applied, but that might cause discharges at the electrodes. Instead, it is recommended to reduce the trapping frequency, since dividing the frequency by two results in a quadrupling of the maximum storable $\mathrm{m} / \mathrm{z}$ value, see Equation 2 and Figure 2.

\section{FFT mode detection}

In most quadrupole ion trap mass spectrometers ions are detected by the use of instability scans [1], [2]. Either the amplitude or the frequency of the trapping waveform is swept. Then ions of different mass-tocharge ratios become unstable and are ejected from the trap through a hole in one of the trap electrodes and registered by a particle detector.

In the past decades different approaches were made to achieve non-destructive ion measurement [3], [4]. In general, the current induced on the trap top and bottom end-cap electrodes by coherent ion movement is measured. Typically, this induced current is detected by low noise amplifiers. The signal amplitude depends on the ion oscillation magnitude, the number of ions and the trap geometry. The resulting current generated by one single ion approximately amounts to a few femtoamperes.

\section{Electronics}

\section{A. Crosstalk Compensation}

Due to the capacitive coupling between the ringelectrode and the end-cap electrodes (see Figure 3), 


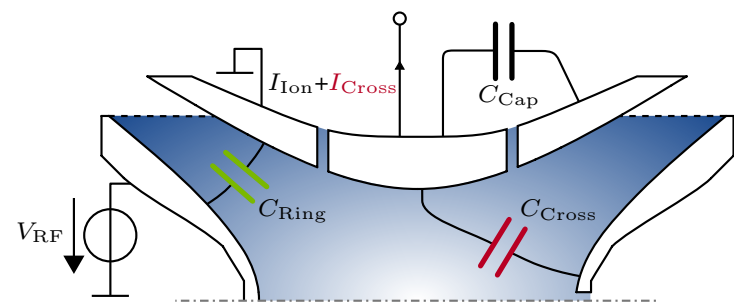

Fig. 3. Close-up of one half of the quadrupole ion trap with segmented end-cap electrode and the parasitic capacitances.

quadrupole ion traps working with induced current detection suffer from a massive RF pickup, typically in the $\mathrm{mA}$ range, exceeding the induced current by many orders of magnitude. This problem can be overcome primarily by three different approaches. Beneficial to all of them is the fact that the ion resonance frequency is always lower than half of the RF trapping frequency, see Equation 1. The first solution (i) involves a resonant tank circuit or narrowband filter, suppressing interference of the RF signal. Another possibility (ii) to reduce the crosstalk signal can be found in splitting up the end-cap electrodes into two segments and using only a small part for the detection of the induced current, thereby reducing the parasitic capacitance $C_{\text {Cross }}$ [3], [4]. However, using "needle"-like detection electrodes entails certain drawbacks, in particular a low sensitivity and the production of higher harmonics in the induced current signal.

Aliman and Glasmachers were the first to present an alternate solution (iii). They used a narrowband compensation signal to neutralize the crosstalk signal, while maintaining a larger inner end-cap electrode [5]

In our setup we are working with non-sinusoidal RF signals (i.a. to enhance mass range), hence we designed a novel broadband compensation circuit to eliminate the crosstalk signal. The overall induced current amplifier design had to meet the following requirements:

- high gain

- wide-bandwidth

- low-noise, as sensitivity is of huge importance for analysis

- $\quad$ keeping virtual ground on the end-cap electrodes, as any offset might cause ion instability

- $\quad$ providing a distortion-free path for ion excitation

Figure 4 shows a simplified schematic of the circuitry. An inverted fraction of the high voltage RF signal is transferred to the capacitance $C_{\mathrm{Comp}}$ by amplifier $\mathrm{OP}_{1}$. Compared to the interference signal $I_{\text {Cross }}$, the current flowing through $C_{\text {Comp }}$ is of opposite phase. Additionally, $C_{\text {Comp }}$ helps to reject any common mode introduced by the compensation circuit. Since the value of $C_{\text {Comp }}$ affects the noise gain of the actual charge amplifier $\left(\mathrm{OP}_{2}\right)$, its dimensioning is a compromise (higher compensation currents $I_{\mathrm{Comp}}$ require either higher output voltages of $\mathrm{OP}_{1}$ which

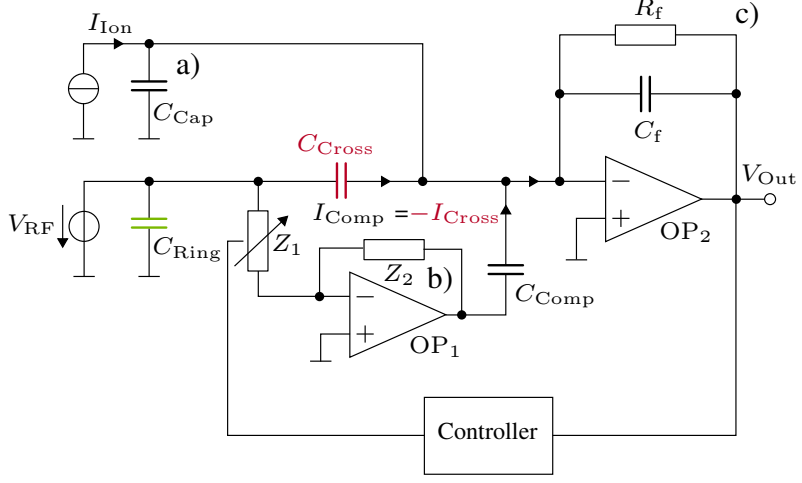

Fig. 4. Simplified schematics of the induced current detection, a) equivalent trap and ion current model b) compensation circuit $c$ ) charge amplifier

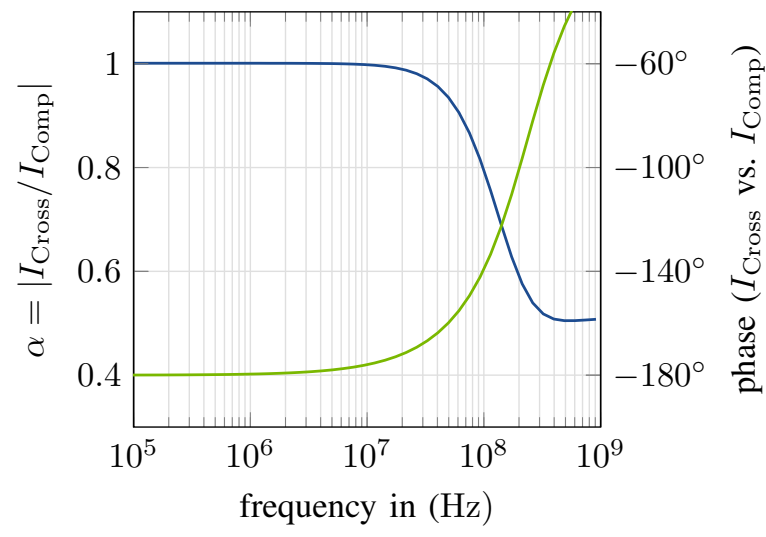

Fig. 5. Simulated frequency response of the compensation circuit, cut-off frequency is located at $>30 \mathrm{MHz}$ to prevent damping of non-sinusoidal shaped signals containing higher harmonics

are limited by the supply voltage, or higher values of $C_{\text {Comp }}$ which leads to worse noise characteristics). The impedances $Z_{1}$ and $Z_{2}$ can be calculated from Equation 5. $Z_{1}$ is adjustable in order to automatically match $I_{\text {comp }}$ to $I_{\text {cross }}$, as the value of $C_{\text {Cross }}$ varies slightly from setup to setup.

$$
\begin{array}{rcc}
I_{\text {Cross }}(t) & = & C_{\text {Cross }} \cdot \frac{\mathrm{d} V_{\mathrm{RF}}(t)}{\mathrm{d} t} \\
I_{\text {Comp }}(t) & = & -\frac{Z_{2}}{Z_{1}} \cdot C_{\text {Comp }} \cdot \frac{\mathrm{d} V_{\mathrm{RF}}(t)}{\mathrm{d} t} \\
0 \stackrel{!}{=} & I_{\text {Cross }}(t)+I_{\mathrm{Comp}}(t) \\
\Rightarrow \alpha= & \frac{C_{\text {Cross }}}{C_{\text {Comp }}}=\frac{Z_{2}}{Z_{1}}
\end{array}
$$

The frequency response of the compensation circuit is displayed in Figure 5. In practice, matching factors $\alpha$ of about $98 \%$ were obtained. Parasitic impedances in the layout and wiring prevent an exact phasing between both currents. Although the new broadband compensation approach adds additional noise (10\% - $20 \%$ increase compared to the previously used narrowband compensation) the complete current detection circuit exhibits an excellent signal-to-noise ratio. A few ions may be detected. Indeed, detection-limits in the pptV range already have been obtained with the narrowband compensation circuit [6], [7]. 


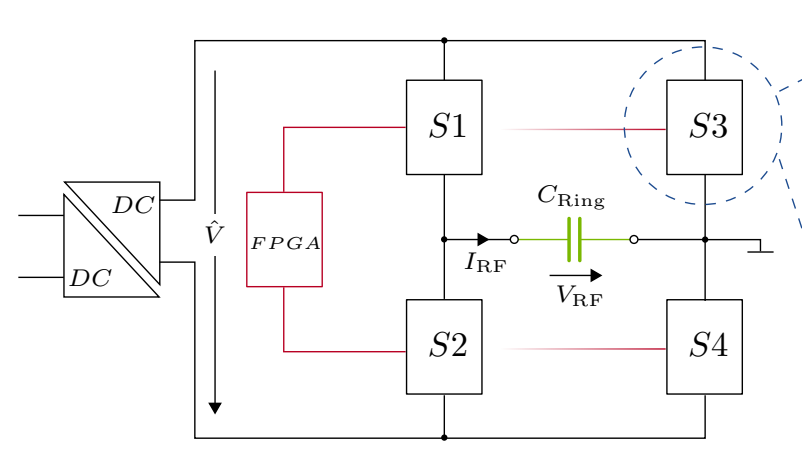

Fig. 6. Full-bridge module with isolated power supply

\section{B. RF Generator}

Most ion traps are operated with sinusoidal trapping waveforms. Typically these are generated by resonant circuits. Power consumption is low but the oscillation/trapping frequency is fixed. Theory proves that any periodic waveform allows for storing ions inside the trap. This has been demonstrated experimentally with rectangular and triangular shaped waveforms and nonFFT-mode operated ion traps [2], [1].

One method to generate high-voltage rectangular waveforms employs half or full-bridge circuits. The concept we chose is displayed in Figure 6. A custom-built isolated DC-DC converter powers a full-bridge. The full-bridge MOSFETs are controlled by isolated gate drivers that allow for switching frequencies up to $1 \mathrm{MHz}$. With the full bridge circuit, only a single power supply is needed. Moreover, several full-bridge modules may be connected in series to create even higher output amplitudes. Since the secondary side of the power supply is kept at floating potential the capacitive coupling needs to be minimized ( $\mathrm{pF}$ range). Apart from offering variable frequency and an arbitrary duty cycle an interval of $V_{\mathrm{RF}}=0 \mathrm{~V}$ may be introduced by simultaneously turning on either both high-side or low-side MOSFETs. This can help to reduce crosstalk currents or may be beneficial to the in-situ electron ionization phase (constant electron energy).

Although the developed compensation circuit offers a wide bandwidth, the high slew-rate of the rectangular waveform can cause undesirable saturation effects on the highly sensitive charge amplifier $\left(\mathrm{OP}_{2}\right)$. Consequently, the slope of the rectangular waveform has to be reduced, especially at higher output amplitudes. Filtering the bridge output signal would introduce additional power loss and cause oscillations. Therefore, since the ion trap ring electrode represents a capacitive load, it is advantageous to control the bridge output current instead of smoothing the voltage.

This can be achieved with an adaptive currentlimiting circuit. Each of the four MOSFETs is still controlled "digitally" but the current flow is actively limited (switched current source), see Figure 7. Transistor $T_{2}$ reduces the gate-source-voltage of $T_{3}$ so that a defined maximum current flows through resistor $R_{S}$. To obtain versatile output currents the apparent solution would be to control the value of $R_{S}$. However, building an isolated voltage-controlled resistor can be

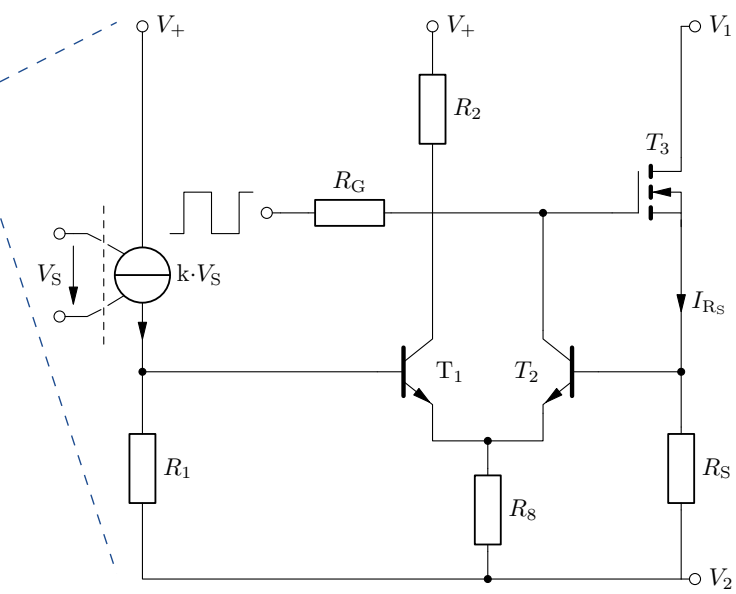

Fig. 7. Simplified schematics of the current limiting circuit for one of the full-bridge MOSFETs (S1-S4 of Figure 6)

challenging. Instead, we decided to control the maximum voltage $V_{R_{S}}$ over $R_{S}$ rather than the resistance itself. This was achieved by adding transistor $T_{1}$ and therefore building a simple differential amplifier. The base potential of both transistors is now regulated with an isolated current source and resistor $R_{1}$. The current can be approximated by

$$
I_{\mathrm{R}_{\mathrm{S}}}=\frac{k V_{S} R_{1}}{R_{S}}+\frac{V_{\text {Offset }}}{R_{S}}
$$

Its minimum value is restricted by the forward voltage of $T_{2}$ and a residual voltage drop on $R_{8}$ denoted by $V_{\text {Offset }}$. Figures 8 a) and b) display the resulting waveforms for different current limits. The current-limiting technique successfully helps to reduce the remaining cross-talk signal at the charge amplifier output, $V_{\text {Out }}$ (see Figure 4), by a factor of 5-10, depending on the slew-rate, see Figures 9 a) and b). With one waveform generator module rectangular-, trapezoidal- or triangular-shaped signals of up to $500 \mathrm{~V}$ and $1 \mathrm{MHz}$ can be generated. If, for example, only $\mathrm{S} 1$ and S4 are switched on for $0 \leq t \leq t_{1}$, than the resulting voltage over $C_{\text {Ring }}$ increases linearly with

$$
V_{\mathrm{RF}}(t)=\frac{I_{\mathrm{R}_{\mathrm{S}}}}{C_{\mathrm{Ring}}} \cdot t+V_{\mathrm{RF}}(0) .
$$

By adjusting the switching times of S1-S4, the switching frequency $f_{\mathrm{RF}}$ and the output voltage of the DC/DC converter $\hat{V}$ the previously mentioned waveforms can be realized.

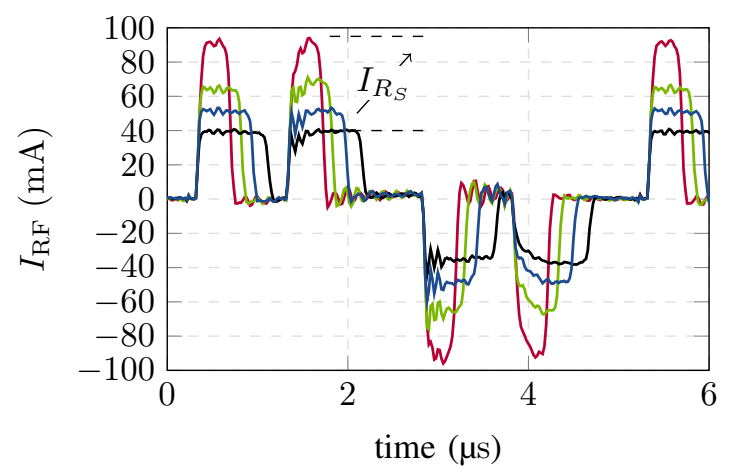

(a) 


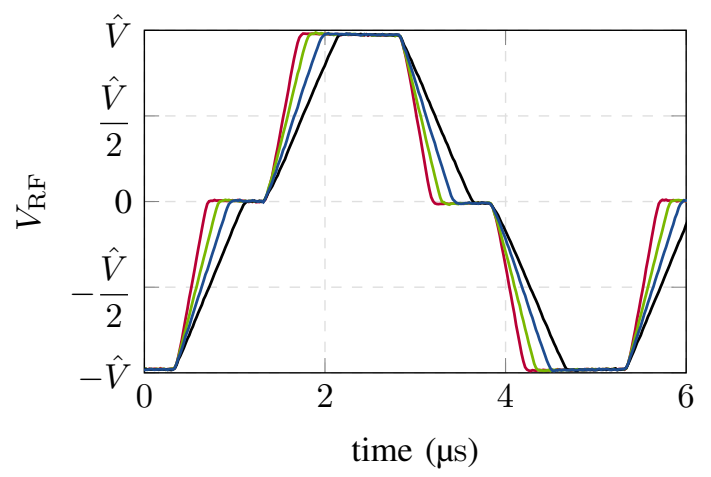

(b)

Fig. 8. Resulting currents a) and voltages b) at the ion trap ring electrode for different current limits at a RF frequency of $200 \mathrm{kHz}$

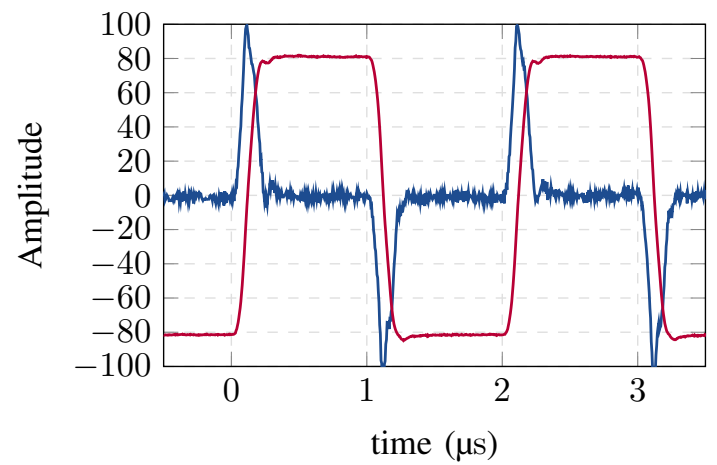

(a)

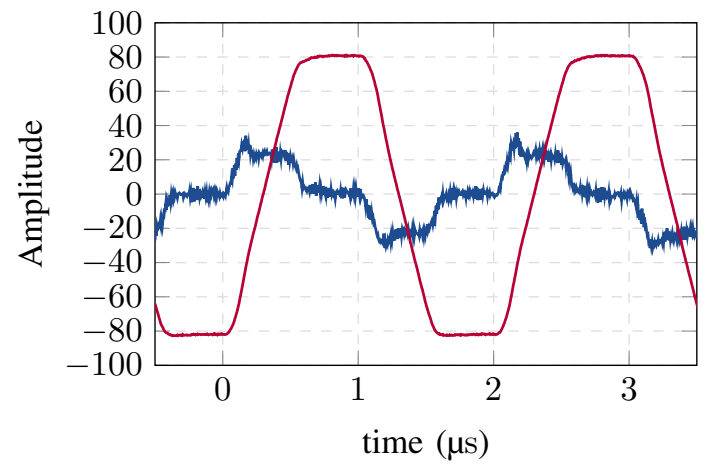

(b)

Fig. 9. Output signal of the charge amplifier $V_{\text {out }}$ (blue, $(\mathrm{mV})$ ) for different slew-rates of the storage waveform (red, (V)), a) rise/fall time $=120 \mathrm{~ns} \mathrm{~b}$ ) rise $/$ fall time $=420 \mathrm{~ns}$

With our mass spectrometer different samples have been examined. Figure 10 displays exemplary spectra of krypton with its typical isotopic distribution obtained with two different storage waveforms.

\section{CONCLUSION}

Operating an ion trap mass spectrometer with a broadband induced current detection is beneficial in terms of resolution, speed and sensitivity. However it requires an efficient, low-noise interference reduction to cancel the RF cross-talk. In our setup this was achieved with the presented compensation network. We are now able to utilize non-sinusoidal trapping waveforms. This has been demonstrated for the trapezoidal case. The modular high voltage generator allows for generation of such storage waveforms with adjustable

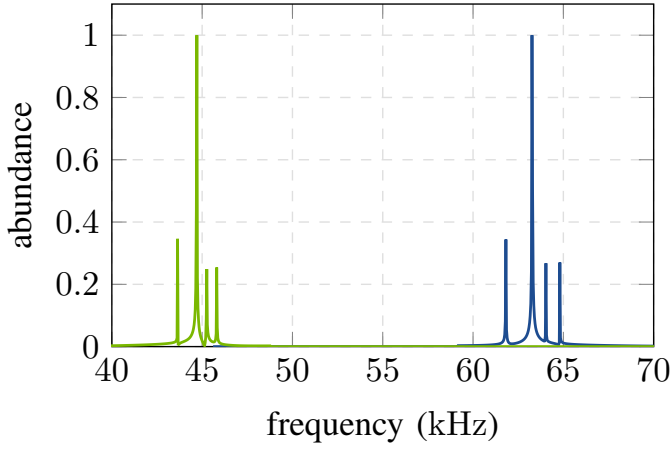

(a)

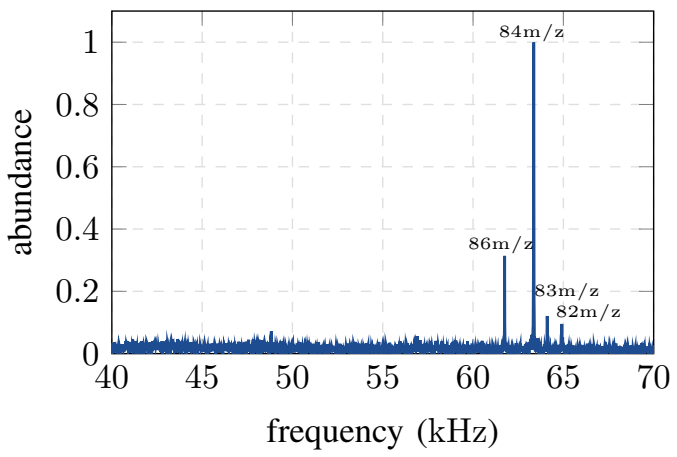

(b)

Fig. 10. Spectrum of krypton with different waveforms a) simulated, b) measured, sinusoidal wave (green) trapezoidal wave (blue)

trapping frequencies. This extends the detectable mass spectrum to the range of heavy molecules $(\mathrm{kDa})$. Furthermore it is possible to create asymmetrical waveforms. This may be used for mass selective storage as it has already been shown for linear quadrupoles [8].

\section{REFERENCES}

[1] C. Xiong, G. Xu, X. Zhou, J. Wang, Y. Tang, R. Chen, W.-P. Peng, H.-C. Chang, and Z. Nie, "The development of charge detection-quadrupole ion trap mass spectrometry driven by rectangular and triangular waves," Analyst, vol. 137, pp. 1199-1204, 2012.

[2] S. Bandelow, G. Marx, and L. Schweikhard, "The stability diagram of the digital ion trap," Int. J. Mass Spectrom., vol. 336, pp. 47 - 52, 2013.

[3] M. Soni, V. Frankevich, M. Nappi, R. E. Santini, J. W. Amy, and R. G. Cooks, "Broad-band fourier transform quadrupole ion trap mass spectrometry," Analytical Chemistry, vol. 68, no. 19, pp. 3314-3320, 1996.

[4] E. R. Badman, G. E. Patterson, J. M. Wells, R. E. Santini, and R. G. Cooks, "Differential non-destructive image current detection in a fourier transform quadrupole ion trap," Journal of Mass Spectrometry, vol. 34, no. 8, pp. 889-894, 1999.

[5] M. Aliman and A. Glasmachers, "A novel electric ion resonance cell design with high signal-to-noise ratio and low distortion for fourier transform mass spectrometry," J. Am. Soc. Mass Spectrom. Chem., vol. 10, no. 10, pp. 1000 - 1007, 1999.

[6] Y. Brachhaeuser, D. Mueller, H. Kersten, K. J. Brockmann, M. A. T. Benter, G. Fedosenko, A. L. R. Reuter, V. Derpmann, and H. Y. Chung, "Micro-plasma based pulsed direct charge transfer stage coupled to a ft-it mass spectrometer," 63th ASMS Conference, St. Louis, USA, 2015.

[7] H. Y. Chung, M. Aliman, G. Fedosenko, A. Laue, R. Reuter, V. Derpmann, L. Gorkhover, and M. Antoni, "Very sensitive real-time inline process mass spectrometer based on $\mathrm{fft}$ ion trap technique," in 27th Annual SEMI Advanced Semiconductor Manufacturing Conference (ASMC), May 2016, pp. 263-266.

[8] F. L. Brancia, B. McCullough, A. Entwistle, J. G. Grossmann, and L. Ding, "Digital asymmetric waveform isolation (dawi) in a digital linear ion trap," J. Am. Soc. Mass Spectrom. Chem., vol. 21 , no. 9 , pp. $1530-1533,2010$ 